\title{
A Novel Iris Segmentation Method for Hand-Held Capture Device
}

\author{
XiaoFu He and PengFei Shi \\ Institute of Image Processing and Pattern Recognition, \\ Shanghai Jiao Tong University, Shanghai 200030, China \\ \{xfhe, pfshi\}asjtu.edu.cn
}

\begin{abstract}
In this paper, a new iris segmentation method for Hand-held capture device is proposed. First, the pupil is binarized using the intensity threshold, then use morphologic method to denoise the eyelashes and eyelids noise. The geometrical method is used to calculate the coordinates of the pupil. Second, the outer (or limbus) boundary is localized using the shrunk image with the Hough transform and modified Canny edge detector in order to reduce computational cost. Third, the eyelids which are constrained to be within the outer boundary are estimated using the polynomial fitting method. The segmentation method was implemented and tested on iris database set which is captured by hand-held optical sensor device. Experimental results show that the proposed algorithm can separate the iris from the surrounding noises with good speed and accuracy.
\end{abstract}

\section{Introduction}

In recent years, iris recognition becomes one of the most reliable biometric technologies. The most important part for an iris recognition system is the iris segmentation. The goal of iris segmentation is to localize the iris from the surrounding noises, such noises include the pupil, the sclera, the eyelids, the eyelashes, the eyebrows and the reflections etc.

Whereas, it is difficult to localize the iris from the surrounding noises, especially for the hand-held capture device. The main reason is that the eyelid or eyelashes usually occlude the iris and the iris capture devices in application are mostly exposed to the natural scene, so the natural illumination or other variant conditions sometimes can greatly influence the iris images and further impact the segmentation result. All these factors will influence the subsequent processing since iris pattern represented improperly will inevitably result in poor recognition performance. To solve this problem, a robust segmentation method should be proposed to remove the influence of all these noises as much as possible.

It is widely accepted that an eye can be modeled by two circles, pupil and limbus, and two parabolas, upper and lower eyelids. In previous segmentation methods, most of which are based on integrodifferential operator or Hough transform. John G. Daugman [1][2][3] proposed an integrodifferential operator for localizing iris regions along with removing the possible eyelid noises. The Daugman system fits the circular contours via gradient ascent together with radial Gaussian. Wildes [4] processed iris segmentation through filtering and voting procedure, which is realized via Hough transform on 
parametric definitions of the iris boundary contours, include pupil, limbus and the eyelids boundaries. W. K. Kong and D. Zhang [5] proposed an accurate iris segmentation method for reflection and eyelash detection. An edge detector is applied to detect separable eyelashes, and intensity variances are used to recognize multiple eyelashes. A threshold and statistical model is proposed to recognize the strong and weak reflection, respectively. Ma et al. [6][7] processed iris segmentation by edge detection and Hough Transform. Eyelids and eyelashes noises were not considered in his method. Huang et al. [8] proposes a new noise-removing approach based on the fusion of edge and region information. Edge information extraction was based on phase congruency. The iris is segmented using edge detection and Hough transform. Using the Hough transform segmentation technique, the circle parameters can be found out in the 3-dimensional parameter spaces. Direct application of the Hough transform for detecting circles and eyelid in images is not practical due to the expensive requirements of 3-dimensional parameter spaces. Space and time complexities are the main concerns in the application of Hough transform for detecting circles. Certainly the smaller of the searching region, the faster of the calculation speed. Moreover, if boundaries of iris pupil and sclera were clearly distinguished, most automatic segmentation methods will be proved to be successful. However, in practical application, it is very difficult to locate the boundary between the iris and the sclera. Difficulties arise from the fact that edge detection will fail to find the edges of the iris border.

So, in this paper, we develop a novel iris segmentation method for hand-held capture device. Since the pupil typically is darker than the iris, the pupil (or inner) boundary is detected using geometrical method together with the intensity threshold, which can reduce computational cost and still gets good results. We use the shrunk image to detect outer circle based on modified Canny edge detector [9][10] together with Hough transform, which has fewer noises, e.g spurious boundaries. The fewer noises are, the smaller of the searching region is. The upper and lower eyelids detection relies on the polynomial fitting method.

The remainder of this paper is organized as follows: Section 2 provides a description of the proposed method for pupil localization. Section 3 introduces the outer boundary detection. Eyelids, eyelashes and reflection detection are also given in this section. Section 4 reports experiments and results. Section 5 concludes this paper.

\section{Pupil Localization}

The iris is an annular part between the pupil (or inner) and the outer (or limbus) boundary. Both of them can approximately be taken as circles. Using the iris prior-knowledge, we first roughly determine the iris region in the original image and use intensity threshold to binarize the iris together with morphological operations. Then use the geometrical method to exactly calculate the parameters of the inner circle in the determined region after edge detection.

\subsection{Rough Localization}

To capture the rich details of iris patterns, an imaging system should resolve a minimum of 70 pixels in iris radius [3]. In most deployments of these algorithms to 
date, the resolved iris radius has typically been 80 to 130 pixels, though some companies have minute differences. So we can use the prior-knowledge to roughly locate the iris region. This will reduce the region for subsequent processing, which results in lower computational cost. Then we use intensity threshold to binarize the iris.

\subsection{Edge Detection}

From the above processing, we usually get a noised binary image, especially the eyelashes and top eyelid, since the intensity of the eyelashes and top eyelid are similar to the pupil in hand-held captured images. All these may affect the subsequent processing, so we use morphological operations to exclude unnecessary regions especially the eyelashes and eyelid in order to get a connected adjacent region. Then we use Sobel operator to extract the edges.

\subsection{Pupil Localization}

The geometrical method is used to locate the circle. We proposed that two perpendicular lines L1 and L2 intersect at P which is the inner of the circle. From the bottom point $A$, we draw several horizontal lines with interval $\triangle$. We then get two point sets A0 $\{\mathrm{A} 10, \mathrm{~A} 20, \mathrm{~A} 30 \ldots\}$ and $\mathrm{A} 1\{\mathrm{~A} 11, \mathrm{~A} 21, \mathrm{~A} 31 \ldots\}$ from these horizontal lines which intersect the circle, as shown in the diagram in Fig.1. Then use three points $\mathrm{a} 0$, a1 and A to calculate the radius and the center coordinates of the circle, since three points which are not on the same line can determine a circumcircle, where a 0 and $\mathrm{a} 1$ comes from point set $\mathrm{A} 0$ and $\mathrm{A} 1$ respectively.

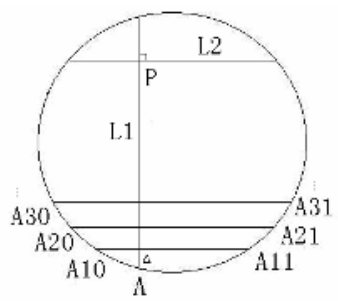

(a)

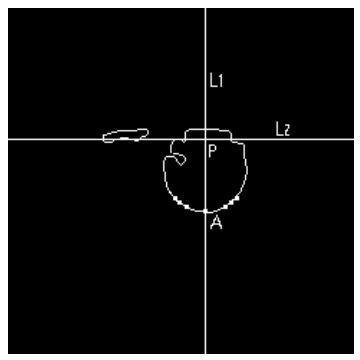

(b)

Fig. 1. Circle localization. (a) Diagram. (b) Point sets overlaid on iris edge image.

We project the binary image in the vertical and horizontal direction to approximately estimate the centroid $\mathrm{P}\left(x_{p}, y_{p}\right)$, which is usually not the center of the pupil. But at least we can see that $\mathrm{P}\left(x_{p}, y_{p}\right)$ is the inner of the pupil since the pupil is usually darker than other areas. We draw two perpendicular lines L1 and L2 through the inner point $\mathrm{P}\left(x_{p}, y_{p}\right)$, shown in Fig.1 (b). Then we get the lowest point A. From the point A, we draw several horizontal lines with interval $\triangle$ which result in two point sets. We use these two point sets and the bottom point $\mathrm{A}$ to calculate the radius and the center of the 
circle and get the average value of the radius and the center of the circle. The result of the pupil localization can be seen in Fig. 2 .

It is difficult to separate the pupil from the surrounding noises especially captured by Hand-held capture device. Since the pupil is partly occluded by the top eyelid and eyelashes. We use the bottom point A of the pupil as the reference point because the bottom point is insensitive to the pupil dilation and not affected by the top eyelid or eyelashes. We have found empirically that the bottom 3 or 4 lines with interval 3 pixels are enough to locate the pupil exactly.

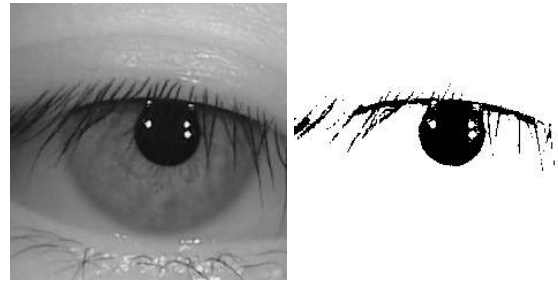

(a)

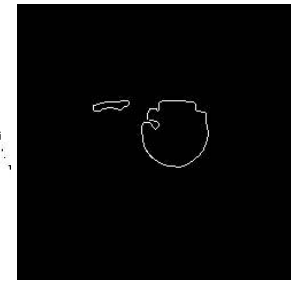

(c)

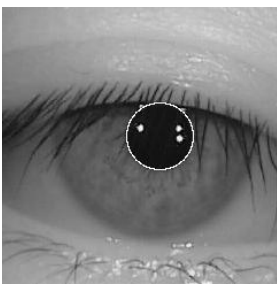

(d)

Fig. 2. Pupil localization. (a) Source image. (b) Binary image. (c) Edge image after morphological operation and deleting small region. (d) Localized image.

\section{Outer Boundary and Non-iris Areas Detection}

\subsection{Outer Boundary Detection}

It is difficult to locate the outer boundary from the surrounding noises when there is little contrast between iris and sclera regions, especially the eyelids or eyelashes usually occlude the iris. So, Hough transform is used to detect outer boundary which is a standard machine vision technique for fitting simple contour models to images. Since space and time complexities are the main concerns in the application of Hough transform. In order to reduce the searching region we use the shrunk image to detect outer boundary together with modified Canny edge operator [9][10]. The output edge map is similar but has fewer noises, e.g spurious boundaries, using the improved Canny edge detector.

First, the image is shrunk with the rate of $30 \%$. Then the shrunk image is filtered with modified Canny edge detector that is tuned in near vertical orientation since even in the face of occluding eyelids or eyelashes, the left and the right portions of the limbus should be clearly visible and oriented near the vertical. Whereas the detected edge usually is a noised image, especially affected by the eyelashes, shown in Fig.3(b). So we cut the top eyelid region and exclude pupil area, where top eyelid region is estimated through the pupil position, shown in Fig.3(c). Then we use 8-directional connection method to further exclude unnecessary edges using the certain threshold in order to save the connected adjacent edges, shown in Fig.3 (d). Second, the radius and the center coordinates of the outer circle are calculated using Hough transform on the denoised edge map, shown in Fig.3 (e). 


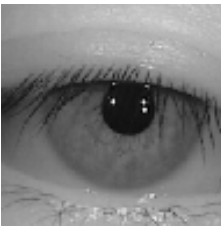

(a)

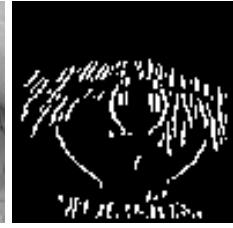

(b)

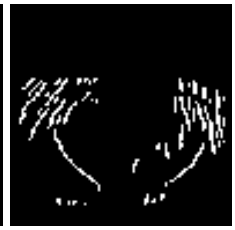

(c)

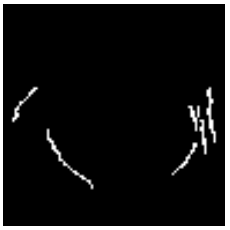

(d)

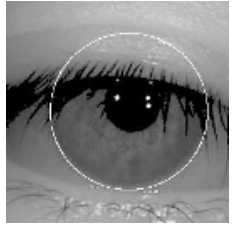

(e)

Fig. 3. Outer boundary detection. (a) Shrunk image. (b) Edge image. (c) Exclude top eyelid and pupil area. (d) Denoised edge image. (e) Outer circle localization result.

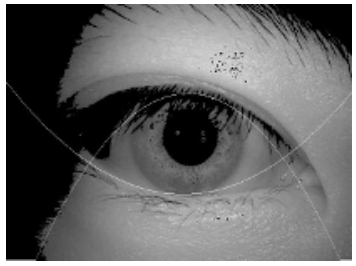

(a)

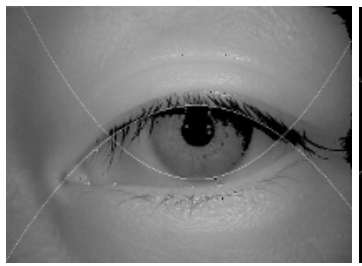

(b)

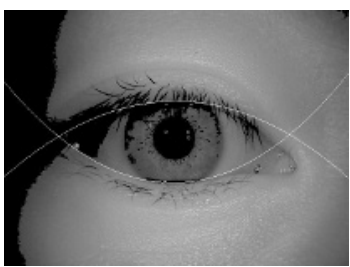

(c)

Fig. 4. Non-iris areas detection samples

\subsection{Eyelids, Eyelashes and Reflection Detection}

If the eyelids occlude part of the iris, then only that portion of the image below the upper eyelid and above the lower eyelid should be included. Here, we use least-squares sense to fit the parabola of eyelid together with modified Canny edge detector, i.e finds the coefficients of a polynomial $\mathrm{P}(\mathrm{X})$ of degree $\mathrm{N}$ that fits the data, $\mathrm{P}(\mathrm{X}(\mathrm{I}))=\mathrm{Y}(\mathrm{I})$ in a least-squares sense. Moreover, we additionally constrained the detected boundaries to be within the outer circle and above the pupil for upper eyelid and below the pupil for lower eyelid, respectively. Since the detected upper eyelid edge map is usually affected by the eyelashes, we use 8-directional connection method to further exclude unnecessary edges using the certain threshold in order to save the connected adjacent edges and exclude pupil areas.

Since the iris especially captured by hand-held capture device has been corrupted by the occlusion of the eyelashes, it is necessary to detect them as much as possible. We can see from Figure 3 (b) that the eyelashes area can be got from the binary image of the eye, so we detect the eyelashes areas using intensity threshold with a proper value. Since the reflection spots in most cases are much lighter than other part of the iris, it is also an effective way to detect them just by a certain threshold. The eyelids, eyelashes and reflection detection result can be seen from Fig.4.

\section{Experimental Results}

In this work, we use Hand-held captured iris database set, which comes from the National Laboratory of Pattern Recognition (NLPR) in China [11] collected by the 


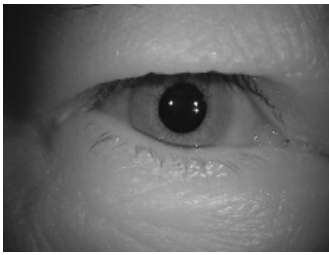

(a)

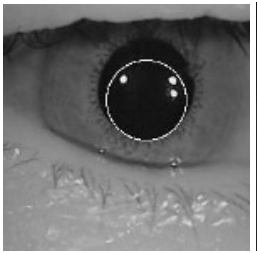

(b)

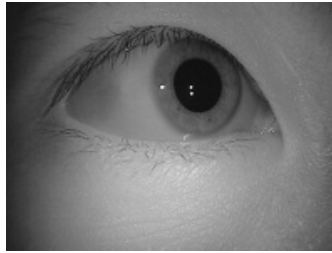

(c)

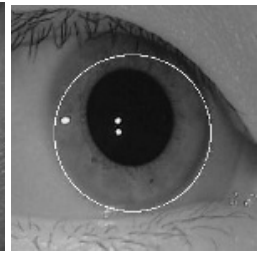

(d)

Fig. 5. Failure localization samples. (a) Source image. (b) Pupil localization failure. (c) Source image. (d) Outer boundary detection failure.

Institute of Automation of the Chinese Academy of Science. The database set includes 1200 grayscale iris images from 60 eyes (hence 60 classes). For each eye, 20 images are captured in one session using the hand-held optical sensor. Image resolution is $640 \times 480$ and the distance between the device and the user is about $4-5 \mathrm{~cm}$.

The proposed methods have been implemented using Matlab 6.5 on a PC with Intel Pentium III $864 \mathrm{MHz}$ processor and $128 \mathrm{MB}$ system memory. We evaluated the success rate for the proposed methods using above iris database set for finding the inner and outer boundary. The correct segmentation rate can reach approximately $97 \%$, only a few cases cannot be segmented correctly mainly due to the capture problem and the eye shape. The failed samples are shown in Fig.5. Moreover, we compared the time consuming of pupil localization with traditional Hough transform. The average time is about 0.7 seconds, whereas is about 76 seconds with traditional Hough method.

In general, under the same conditions and using the same iris database, the pupil localization of the proposed method speeds up with good localization accuracy. Whereas, in the case of that position of the iris is not correct or the pupil is distorted, our method will fail to localize the iris.

\section{Conclusion}

In this paper, a new iris segmentation method for hand-held capture device is presented. Pupil (or inner) boundary is detected using the geometrical method. In order to reduce computational cost, the shrunk image is used to detect outer boundary using Hough transform together with modified Canny edge detector. The polynomial fitting method is used to fit the parabola of eyelid together with modified Canny edge detector. Experimental results have illustrated the encouraging performance of the current method in accuracy.

In the future work, we will conduct experiments on a large number of iris databases in various environments for the proposed method to be more stable and reliable.

\section{Acknowledgements}

This work is funded by NNSF (No.60427002). Portions of the research in this paper use the CASIA iris image database collected by Institute of Automation, Chinese Academy of Sciences. 


\section{References}

1. J. G. Daugman. High Confidence Visual Recognition of persons by a Test of Statistical Independence, IEEE Transaction on Pattern Analysis and Machine Intelligence, 15(11),(1993) 1148-1160

2. J.G. Daugman. The importance of being random: Statistical principles of iris recognition. Pattern Recognition,36(2), (2003) 279-291

3. J. G. Daugman. How iris recognition works. IEEE Transactions on Circuits and Systems for Video Technology,14(1), (2004) 21-30

4. R.Wildes. Iris recognition: An emerging biometric technology. Proceedings of the IEEE, 85(9), (1997) 1348-1363

5. W. K. Kong and D. Zhang. Detecting eyelash and reflection for accurate iris segmentation. International Journal of Pattern Recognition and Artificial Intelligence, 17(6),(2003)1025-1034

6. L. Ma, T. Tan, Y. Wang, D. Zhang. Efficient iris recognition by characterizing key local variations. IEEE Transactions on Image Processing,13(6),(2004) 739-750

7. L. Ma, T. Tan, Y. Wang, D. Zhang. Personal Recognition Based on Iris Texture Analysis. IEEE Transaction on Pattern Analysis and Machine Intelligence,25(12),( 2003) 1519-1533

8. J.Z. Huang, Y.H. Wang, T. Tan, et al. A new iris segmentation method for recognition. Proceedings of the 17th International Conference on Pattern Recognition.Vol.3,(2004) 554-557

9. Fleck, M.M. Some defects in finite-difference edge finders. IEEE Transactions on Pattern Analysis and Machine Intelligence, 14(3),(1992)337-345

10. Canny, J.,A Computational Approach to Edge Detection, IEEE Transaction on Pattern Analysis and Machine Intelligence, 8,(1986)679-714

11. CASIA Iris Image Database, http://www.sinobiometrics.com 